\section{Ranibizumab for retinal angiomatous proliferation in age-related macular degeneration}

\begin{abstract}
Purpose To assess the 1-year functional outcome and to evaluate the morphological changes after intravitreal injections of ranibizumab in eyes affected with retinal angiomatous proliferation (RAP) due to age-related macular degeneration (AMD). Methods A prospective, non-randomized, interventional study was conducted on $\mathbf{2 6}$ consecutive patients with newly diagnosed RAP. All eyes were treatment naive and were randomized to receive intravitreal injections of ranibizumab for a 12-month period. After the first three monthly injections, re-treatment was performed in case of best-corrected visual acuity (BCVA) loss of at least five letters associated with fluid within the macula, central macular thickness (CMT) increase of at least $100 \mu \mathrm{m}$, and/or persistence of fluid within the macula as evaluated by optical coherence tomography, new onset macular haemorrhages, persistence of leakage from the lesions on fluorescein angiography.

Results All patients completed the 12-month follow-up: 25 of the 29 treated eyes (86.2\%) were stabilized, with a loss of less than $\mathbf{1 5}$ letters. Nineteen eyes $(65.5 \%)$ maintained or improved their BCVA, and three eyes (10.3\%) gained three lines or more. Overall, mean BCVA remained stable at the 12-month follow-up ( -0.07 letters; $P>0.05$ ). Mean CMT significantly decreased from $386 \pm 147$ to $216 \pm 74 \mu \mathrm{m}$ at the 12-month follow-up. No significant adverse events were observed during the study. The mean number of injections was $5.8 \pm 1.7$ during the follow-up period.

Conclusion The 1-year follow-up outcomes in our series suggest that ranibizumab is an effective treatment for RAP in AMD, allowing stabilization of BCVA and reduction of CMT.
\end{abstract}

K Atmani, M Voigt, V Le Tien, G Querques, G Coscas, G Soubrane and EH Souied

Eye (2010) 24, 1193-1198; doi:10.1038/eye.2010.9; published online 12 February 2010

Keywords: age-related macular degeneration; retinal angiomatous proliferation; chorioretinal anastomosis; type 3 neovascularization; intravitreal ranibizumab

\section{Introduction}

Retinal angiomatous proliferation (RAP), also known as a retinal choroidal anastomosis (RCA) or type 3 neovascularization, is a distinct form of exudative age-related macular degeneration (AMD) characterized by an abnormal communication between the choroidal and the retinal vasculatures. The pathogenesis of this peculiar entity, which was first described in 1992 as a retinal angiomatous lesion associated with drusen, causing retinal pigment epithelium detachments (PEDs), ${ }^{1}$ and in 1995 as an RCA causing PEDs, ${ }^{2,3}$ still remains controversial. In 2001, Yannuzzi et al suggested that an angiomatous proliferation originating from the deep retinal layers extends posteriorly to anastomose with a sub-retinal pigment epithelium (RPE) neovascularization (type 1). ${ }^{4}$ In 2003, on the basis of clinical and angiographic observations, Gass et $a l^{5}$ postulated that the initial neovascular event for RAP is an occult type 1 neovascularization extending anteriorly to form a sub-retinal (type 2) 'piggyback' neovascularization, resulting ultimately in an RCA. The term 'type 3 neovascularization' proposed by Freund et $a l^{6}$ makes no reference to pathogenesis and could be helpful to distinguish this lesion from the two other types of neovascularization, the well-defined type 2 choroidal neovascularization $(\mathrm{CNV})$ and the occult type 1 (CNV). ${ }^{7,8}$ Until the anti-VEGF era, treatment of RAP has been disappointing, with
Department of

Ophthalmology, Centre Hospitalier Intercommunal de Créteil, University of Paris XII, Créteil, France

Correspondence: G Querques, Department of Ophthalmology, Centre Hospitalier Intercommunal de Créteil, University of Paris XII, 40 avenue de Verdun, Créteil 94000,

France

Tel: + 3301451752 22; Fax: + 330145175227 . E-mail: giuseppe.querques@ hotmail.it

Received: 18 August 2009 Accepted in revised form: 11 January 2010 Published online: 12 February 2010 
poor anatomical and functional outcomes. Direct laser photocoagulation of the vascular lesion, laser photocoagulation of the feeder vessel, photodynamic therapy, and transpupillary thermotherapy rarely allow the anatomical closure of the lesion, especially once the vascular complex is well established. ${ }^{9}$ Promising outcomes are expected from the use of anti-VEGF drugs, as shown by the short-term results of intravitreal pegaptanib sodium, ${ }^{10}$ ranibizumab, ${ }^{11,12}$ and bevacizumab $^{13}$ in the treatment of these lesions.

The purpose of this study was to evaluate prospectively the effectiveness of ranibizumab in eyes affected with RAP because of AMD.

\section{Materials and methods}

A total of 26 consecutive patients with newly diagnosed RAP due to AMD were treated with intravitreal ranibizumab and prospectively followed up. All patients underwent a complete ophthalmologic examination at baseline, including measurement of best-corrected visual acuity (BCVA) using standard early treatment of diabetic retinopathy study charts, fundus biomicroscopy, fluorescein angiography (FA), infracyanine green angiography (ICG), and optical coherence tomography (OCT). Diagnosis of RAP was based on FA features confirmed by ICG and OCT. Exclusion criteria were BCVA lower than 20/320, any earlier treatment (such as laser photocoagulation, photodynamic therapy, intravitreal injections of steroids, or anti-VEGF), high myopia ( $>6$ dioptres), RAP due to causes other than AMD (such as macular telangiectasia, inherited macular dystrophy). During follow-up, all patients were monthly evaluated as regards BCVA, fundus colour photographs and OCT, and, in addition, quarterly, or 'as needed', as regards FA and ICG.

After baseline evaluation, a series of three monthly injections was performed and further treatments were given if any of the following changes applied: BCVA loss of at least five letters associated with fluid within the macula as evaluated by OCT, central macular thickness (CMT) increase of at least $100 \mu \mathrm{m}$, and/or persistence of fluid within the macula as evaluated by OCT, new onset macular haemorrhages, persistence of leakage from the lesions on FA. CMT was measured using Spectralis spectral domain OCT (Spectralis SD-OCT; Heidelberg Engineering, Heidelberg, Germany). CMT was defined as the distance between the inner limiting membrane and the RPE, thus excluding fluid under the RPE.

The primary end point was the proportion of eyes losing on BCVA less than 15 letters ('responders') at months 6 and 12. The secondary end points were the rate of maintenance or gain $\geqslant 0$ letters ('maintainers'), the rate of gain $\geqslant 15$ letters ('gainers'), and the rate of severe visual loss (loss $\geqslant 30$ letters).

Morphological outcomes (cystoid macular oedema (CMO), sub-retinal fluid (SRF), PED) were also analysed. This analysis was based on the results of SD-OCT imaging. The choice of a SD-OCT was dictated by its capability to detect very small amounts of fluid in the retina. Thus, $\mathrm{CMO}$ was defined as presence of retinal cysts within the inner and/or outer neurosensory retina; PED corresponded to the anatomical separation between Bruch's membrane and RPE layer; SRF was a fluid accumulation between neurosensory retina and RPE layer.

Statistical calculations were performed using STATA (StataCorp LP, College Station, TX, USA) $10 \mathrm{MP}$ for MacOs X. Comparisons of mean BCVA (converted to the logarithm of the minimum angle of resolution, LogMAR) and CMT were performed using the ANOVA test. The chosen level of statistical significance was $P<0.05$.

\section{Results}

The study included 29 eyes of 26 patients, 7 men and 19 women. Mean age was 78.2 years (SD 6.7) ranging from 66 to 90 years. All patients completed the 12-month follow-up. All the included lesions were graded as stages 2 and 3 according to Yannuzzi's classification. ${ }^{4}$ There were no episodes of inflammation or severe decrease of vision immediately after an injection. During the study, no ocular or systemic adverse events such as thromboembolic events (cerebrovascular accidents, transient ischaemic attacks, myocardial infarctions, or peripheral vascular disease) were reported. The mean number of injections was $5.8 \pm 1.7$ during the 12-month follow-up period.

\section{Functional changes}

Functional outcomes are summarized in Table 1. Mean BCVA was $0.49 \log$ MAR at baseline, $0.48 \log$ MAR at month $6(P>0.05)$, and $0.53 \log$ MAR at 12 months $(P>0.05)$. A slight improvement $(P>0.05)$ in average BCVA occurred over the first 2 months. After that, mean BCVA improvement slightly regressed $(P>0.05)$, reaching baseline level between months 3 and 10. During the last 2 months of follow-up, average BCVA slightly decreased again $(P>0.05)$. Overall, mean BCVA change was -0.07 letters at month 12 compared with baseline (P>0.05; Figure 1).

At month 6, 25 of 29 eyes (86\%) were stabilized with a loss of less than three lines of BCVA ('responders'). Among these 25 eyes, 20 eyes (69\%) maintained or improved their BCVA, and 3 eyes $(10 \%)$ gained at least three lines. One eye $(3 \%)$ presented a severe visual loss 
Table 1 Mean BCVA, maintenance, gain and loss at baseline and months 3, 6, 9, and 12

\begin{tabular}{lccccc}
\hline & Baseline & Month 3 & Month 6 & Month 9 & Month 12 \\
\hline Mean BCVA (logMAR) & $0.493 \pm 0.284$ & $0.486 \pm 0.351$ & $0.479 \pm 0.351$ & $0.476 \pm 0.343$ & $0.503 \pm 0.396$ \\
Loss $<15$ letters, $n(\%)$ & - & $25(86)$ & $25(86)$ & $25(86)$ & $25(86)$ \\
Maintenance or gain $\geqslant 0$ letters, $n(\%)$ & - & $19(66)$ & $20(69)$ & $21(72)$ & $21(72)$ \\
Gain $\geqslant 15$ letters, $n(\%)$ & - & $2(7)$ & $3(10)$ & $3(10)$ & $3(10)$ \\
Loss $\geqslant 15$ letters, $n(\%)$ & - & $4(14)$ & $4(14)$ & $4(14)$ & $4(14)$ \\
Loss $\geqslant 30$ letters, $n(\%)$ & - & $0(0)$ & $1(3)$ & $1(3)$ & $2(7)$ \\
\hline
\end{tabular}

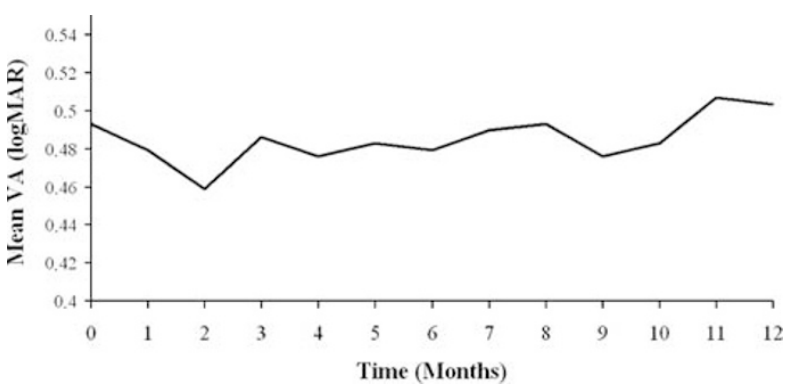

Figure 1 Mean visual acuity (VA) change from baseline until month 12 .

defined as a loss of 30 letters of BCVA or more. At month 12, 25 eyes were 'responders' (86\%), 20 'maintainers' $(69 \%)$, and 3 gainers (10\%). Two eyes (7\%) experienced a severe visual loss, due to the development of a central fibrosis with persistent leakage and exudation.

\section{Morphological changes}

Morphological outcomes are summarized in Table 2. Overall, mean CMT decreased significantly at the 12month follow-up $(P<0.0001)$ from $386 \pm 147$ to $254 \pm 100 \mu \mathrm{m}$ at month $6(P<0.0001)$ and to $217 \pm 74 \mu \mathrm{m}$ at month $12(P=0.03$; Figure 2$)$.

At baseline, CMO was found in 27 of the 29 treated eyes $(93.1 \%)$. It was found in 16 eyes $(55.2 \%)$ at month 6 , and in 14 eyes $(48.3 \%$ ) at month 12 (Figure 3).

Sub-retinal fluid resorption followed the same course. At baseline, OCT showed a SRF in 18 eyes. At months 6 and 12, SRF persisted in five eyes (Figure 3).

Pigment epithelium detachment, which is a step of the natural course of RAP, was found in 25 of 29 eyes (86.2\%) at baseline, and was enough prominent to be clinically detectable on fundus biomicroscopy in eight eyes. PED was detectable in 18 eyes $(62.1 \%)$ at the 6-month follow-up, and in 19 eyes $(65.5 \%)$ at the 12-month follow-up (Figure 3). In one eye, an RPE tear occurred, complicating PED, after three ranibizumab injections and was detected at the 4 month follow-up. This complication had no deleterious impact on visual acuity that remained unchanged.
Table 2 Mean CMT, presence of PED, retinal cysts and SRF, at baseline and months $3,6,9$, and 12

\begin{tabular}{lccccc}
\hline & Baseline & $\begin{array}{c}\text { Month } \\
3\end{array}$ & $\begin{array}{c}\text { Month } \\
6\end{array}$ & $\begin{array}{c}\text { Month } \\
9\end{array}$ & $\begin{array}{c}\text { Month } \\
12\end{array}$ \\
\hline Mean CMT $(\mu \mathrm{m})$ & $386 \pm 147$ & $234 \pm 87$ & $254 \pm 100$ & $224 \pm 51$ & $217 \pm 74$ \\
PED, $n(\%)$ & $25(86)$ & $19(66)$ & $18(62)$ & $19(66)$ & $19(66)$ \\
Retinal cysts, $n(\%)$ & $27(93)$ & $12(41)$ & $16(55)$ & $15(52)$ & $14(48)$ \\
SRF, $n(\%)$ & $18(62)$ & $7(24)$ & $5(17)$ & $5(17)$ & $5(17)$ \\
RPE tear, $n(\%)$ & $0(0)$ & $0(0)$ & $1(3)$ & $1(3)$ & $1(3)$ \\
\hline
\end{tabular}

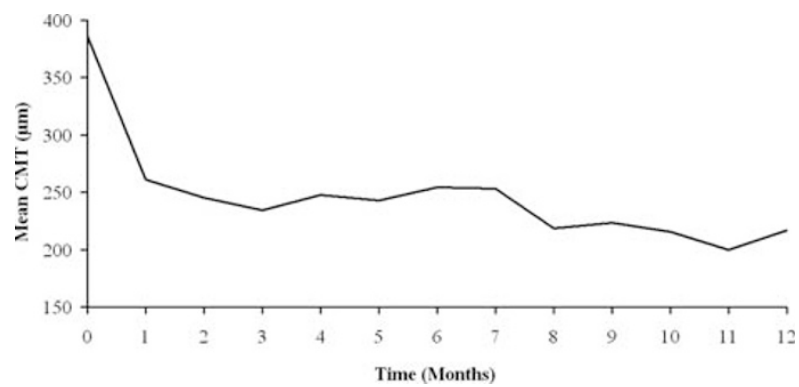

Figure 2 Mean central macular thickness (CMT) change from baseline until month 12 .

At month 12, the rate of CMO, SRF, and PED was reduced respectively by a proportion of 24,48 , and $72 \%$ compared with baseline.

\section{Discussion}

Retinal angiomatous proliferation is a distinct and quite frequent phenotype of AMD, accounting for $8-15 \%$ of newly diagnosed cases. ${ }^{3,14,15}$ This peculiar form of AMD is characterized by a high and cumulative risk of bilateralization, estimated to be as high as $40 \%$ after 1 year, $56 \%$ after 2 years, and $100 \%$ after 3 years. ${ }^{16}$ ICG, by improving the imaging of choroidal circulation, is an invaluable diagnostic tool in such cases, showing typically a late focal area of intense hyperfluorescence described as a hot spot.

The exact pathogenesis of this AMD subtype remains unknown. Some authors proposed that the lesion would 
Presence of PED, CME and SRF

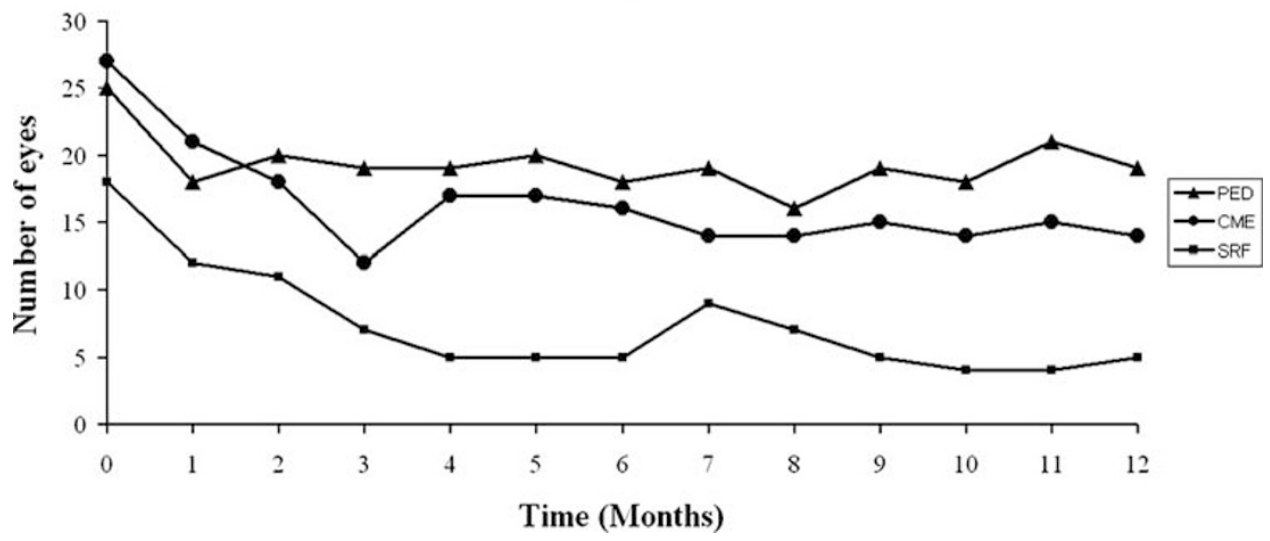

Figure 3 Eyes showing pigment epithelium detachment (PED), cystoid macular oedema (CMO), and sub-retinal fluid (SRF) from baseline until month 12 .

start in the choroid and gain access to the retina through breaks in the Bruch's membrane, ${ }^{2,3,5,6}$ whereas others suggest that the lesion originates from the deep retinal layers. ${ }^{4,17}$

Targeting the VEGF is a reasonable approach to treat RAP. Type 1 and type 2 CNVs are due to a modification in the balance of growth factor secreted by RPE and a similar mechanism could account for type 3 neovascularization in AMD. ${ }^{18}$

The results of this study show that at month $12,86 \%$ of eyes lost less than 15 letters, and $10 \%$ of eyes gained 15 letters or more. These data may be compared with those obtained in other studies such as the MARINA study ${ }^{19}$ for either minimally classic or occult type $1 \mathrm{CNV}$ in AMD, or the PrONTO study in which the therapeutic scheme is globally similar to the one applied on our case series. In the MARINA Trial, patients with AMD with either minimally classic or occult (with no classic lesions) $\mathrm{CNV}$ were randomized to receive monthly intravitreal injections of ranibizumab (either 0.3 or $0.5 \mathrm{mg}$ ) or sham injections. At month 12, in the subgroup treated with ranibizumab $0.5 \mathrm{mg}, 94.6 \%$ of eyes lost less than 15 letters and $33.8 \%$ of eyes improved their visual acuity by 15 letters or more. ${ }^{19}$

In the PrONTO Study, a prospective non-randomized clinical study, patients affected with neovascular AMD were scheduled to receive three consecutive monthly intravitreal injections of ranibizumab $(0.5 \mathrm{mg})$.

Thereafter, re-treatment with ranibizumab was performed if any criterion was fulfilled as a loss of five letters or more or an increase in OCT-CRT. ${ }^{20}$ At month 12, $95 \%$ of eyes (38 of 40) lost less than three lines and among them $35 \%$ gained at least three lines. It is noteworthy that in our study, mean BCVA slightly decreased at month 12 compared with baseline; these data are in contradiction

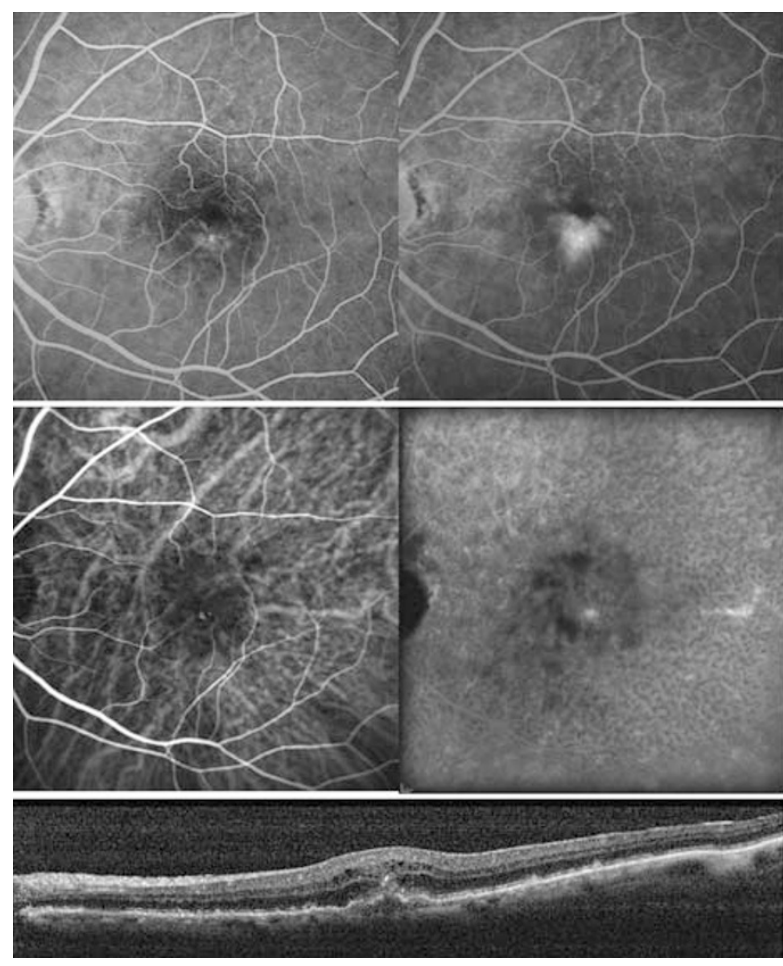

Figure 4 An 87-year-old female patient with early onset visual acuity impairment and metamorphopsia. Baseline examination (best-corrected visual acuity $=20 / 40$ ). Fluorescein angiography (FA) frames reveal an infero-macular hyperfluorescent area at early phase (upper left panel), showing an intense leakage at the late phase frames. Infracyanine green angiography (ICG) frames reveal three hyperfluorescent points (middle left panel) at the early phase that merge into a unique hot spot (middle right panel) in the late phase. Optical coherence tomography (OCT) horizontal scan (bottom panel) showing cystoid spaces within the neurosensory retina. Note a small pigment epithelium detachment with a disruption of the retinal pigment epithelium layer. 

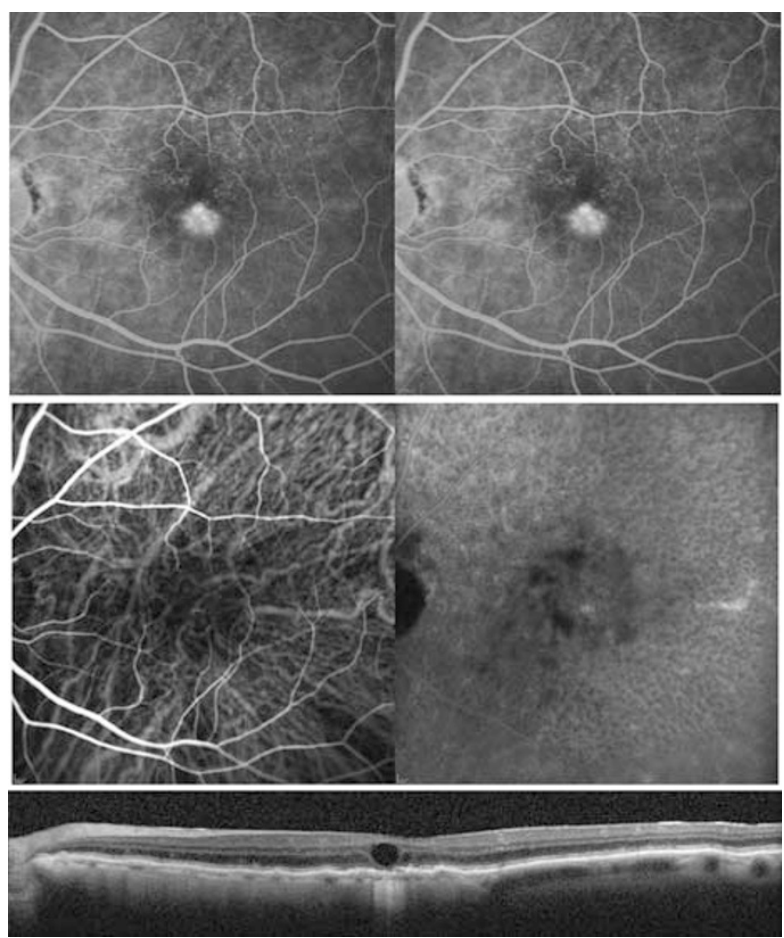

Figure 5 Month 12 examination (best-corrected visual acuity $=20 / 40$ ). After five intravitreal ranibizumab injections, visual acuity is maintained. Fluorescein angiography (FA) frames (upper left and right panels) showing less intense leakage. Infracyanine green angiography (ICG) frames (middle left and right panels) showing a smaller but still active lesion (late hot spot). Optical coherence tomography (OCT) horizontal scan (bottom panel) showing reduced central macular thickness and the resolution of pigment epithelium detachment. A large cystoid space persists with an associated atrophic change.

with the ascending BVCA curves of MARINA or PrONTO studies. One explanation could be the relatively good baseline BCVA (0.49 logMAR corresponding approximately to 20/63 Snellen) in our series, which gives little place to further improvement.

One limit of our study is the absence of a control arm. Another limit is the lack of comparison between anti-VEGF therapy and other therapies. However, medical literature gives us some indication about the efficiency of treatments on RAP. Various treatments for RAP provide poor visual outcomes as shown by conventional laser photocoagulation, ${ }^{9}$ transpupillary thermotherapy, ${ }^{21}$ surgical ablation, ${ }^{22,23}$ or PDT alone. ${ }^{24,25}$ Combined therapies may offer better results, especially in case of PDT combined with intravitreal triamcinolone. ${ }^{26}$

Finally, the size of our series was limited to 29 eyes of 26 patients. However, the follow-up in this series is one of the longest (12 months) and all eyes were treatment naive.

The results of our study appear to be encouraging but remain weaker than those observed in MARINA or
PrONTO studies. Intravitreal ranibizumab seems to be insufficient to give a definitive occlusion of the high blood-flow complex, represented by the RCA, when well established (Figures 4 and 5). Conversely, intravitreal ranibizumab takes effect on vascular hyperpermeability, strongly reducing the fluid accumulation in and/or beneath the neurosensory retina (Figures 4 and 5). Its effect on PED is less obvious, probably because PED, which is frequently associated with this phenotype, is not only due to exudation but may be related to changes in resistance to water flow in the Bruch's membrane. ${ }^{18}$ In our series the PED was detectable in $86 \%$ of eyes at baseline and in $66 \%$ of eyes at month 12 .

Retinal angiomatous proliferation is known to be a severe subtype of AMD, characterized by a high rate of recurrence. Intravitreal injections of ranibizumab may contribute to improve the prognosis of this disease. In our study, after 12 months, intravitreal ranibizumab allowed to stabilize BCVA in $86 \%$ of the 29 treated eyes, which lost less than 15 letters at month 12 , whereas 3 eyes (10\%) gained 15 letters or more. Similarly, CMT decreased by $169 \mu \mathrm{m}$ at month 12 .

These results are to be confirmed by additional randomized studies, including a larger number of eyes. In conclusion, intravitreal ranibizumab appears as a valid therapeutic option in RAP due to AMD.

\section{Conflict of interest}

The authors declare no conflict of interest.

\section{References}

1 Hartnett ME, Weiter JJ, Gardts A, Jalkh AE. Classification of retinal pigment epithelium detachments associated with drusen. Graefes Arch Clin Exp Ophthalmol 1992; 230: 11-19.

2 Kuhn D, Meunier I, Soubrane G, Coscas G. Imaging of chorioretinal anastomoses in vascularized retinal pigment epithelium detachments. Arch Ophthalmol 1995; 113: 1392-1398.

3 Schneider U, Gelisken F, Kreissig I. Retinal choroidal anastomosis in classic choroidal neovascularization demonstrated by indocyanine green angiography. Acta Ophthalmol Scand 1995; 73: 450-452.

4 Yannuzzi LA, Negrão S, Iida T, Carvalho C, RodriguezColeman $\mathrm{H}$, Slakter $\mathrm{J}$ et al. Retinal angiomatous proliferation in age-related macular degeneration. Retina 2001; 21: 416-434.

5 Gass JD, Agarwal A, Lavina AM, Tawansy KA. Focal inner retinal hemorrhages in patients with drusen: an early sign of occult choroidal neovascularization and chorioretinal anastomosis. Retina 2003; 23: 741-751.

6 Freund KB, Ho IV, Barbazetto IA, Laud K, Ferrara D, Matsumoto $Y$ et al. Type 3 neovascularization: the expanded spectrum of retinal angiomatous proliferation. Retina 2008; 28: 201-211. 
7 Gass JD. Serous retinal pigment epithelial detachment with a notch. A sign of occult choroidal neovascularization. Retina 1984; 4: 205-220.

8 Soubrane G, Coscas G. Natural history of occult subretinal new vessels in age-related macular degeneration. Doc Ophthalmol Proc Ser 1987; 50: 219-222.

9 Bottoni F, Massacesi A, Cigada M, Viola F, Musicco I, Staurenghi G. Treatment of retinal angiomatous proliferation in age-related macular degeneration: a series of 104 cases of retinal angiomatous proliferation. Arch Ophthalmol 2005; 123: 1644-1650.

10 Mahmood S, Kumar N, Lenfestey PM, Murjaneh S, Heimann H, Harding SP. Early response of retinal angiomatous proliferation treated with intravitreal pegaptanib: a retrospective review. Eye 2009; 23: 530-535.

11 Lai TY, Chan WM, Liu DT, Lam DS. Ranibizumab for retinal angiomatous proliferation in neovascular age-related macular degeneration. Graefes Arch Clin Exp Ophthalmol 2007; 245: 1877-1880.

12 Konstantinidis L, Mameletzi E, Mantel I, Pournaras JA, Zografos L, Ambresin A. Intravitreal ranibizumab (Lucentis(R)) in the treatment of retinal angiomatous proliferation (RAP). Graefes Arch Clin Exp Ophthalmol 2009; 247: 1165-1171.

13 Meyerle CB, Freund KB, Iturralde D, Spaide RF, Sorenson JA, Slakter JS et al. Intravitreal bevacizumab (Avastin) for retinal angiomatous proliferation. Retina 2007; 27: 451-457.

14 Cohen SY, Creuzot-Garcher C, Darmon J, Desmettre T, Korobelnik JF, Levrat $\mathrm{F}$ et al. Types of choroidal neovascularisation in newly diagnosed exudative age-related macular degeneration. Br J Ophthalmol 2007; 91: 1173-1176.

15 Leveziel N, Zerbib J, Richard F, Querques G, Morineau G, Fremeaux-Bacchi V et al. Genotype-phenotype correlations for exudative age-related macular degeneration associated with homozygous HTRA1 and CFH genotypes. Invest Ophthalmol Vis Sci 2008; 49: 3090-3094.

16 Gross NE, Aizman A, Brucker A, Klancnik Jr JM, Yannuzzi LA. Nature and risk of neovascularization in the fellow eye of patients with unilateral retinal angiomatous proliferation. Retina 2005; 25: 713-718.

17 Monson DM, Smith JR, Klein ML, Wilson DJ. Clinicopathologic correlation of retinal angiomatous proliferation. Arch Ophthalmol 2008; 126: 1664-1668.

18 Holz FG, Pauleikhoff D, Klein R, Bird AC. Pathogenesis of lesions in late age-related macular disease. Am J Ophthalmol 2004; 137: 504-510.

19 Rosenfeld PJ, Brown DM, Heier JS, Boyer DS, Kaiser PK, Chung CY, et al., MARINA Study Group. Ranibizumab for neovascular age-related macular degeneration. $\mathrm{N} \mathrm{Engl} \mathrm{J} \mathrm{Med}$ 2006; 355: 1419-1431.

20 Fung AE, Lalwani GA, Rosenfeld PJ, Dubovy SR, Michels S, Feuer WJ et al. An optical coherence tomography-guided, variable dosing regimen with intravitreal ranibizumab (Lucentis) for neovascular age-related macular degeneration. Am J Ophthalmol 2007; 143: 566-583.

21 Kuroiwa S, Arai J, Gaun S, Iida T, Yoshimura N. Rapidly progressive scar formation after transpupillary thermotherapy in retinal angiomatous proliferation. Retina 2003; 23: 417-420.

22 Sakimoto S, Gomi F, Sakaguchi H, Tano Y. Recurrent retinal angiomatous proliferation after surgical ablation. $A m \mathrm{~J}$ Ophthalmol 2005; 139: 917-918.

23 Shiragami C, Iida T, Nagayama D, Baba T, Shiraga F. Recurrence after surgical ablation for retinal angiomatous proliferation. Retina 2007; 27: 198-203.

24 Boscia F, Furino C, Sborgia L, Reibaldi M, Sborgia C. Photodynamic therapy for retinal angiomatous proliferations and pigment epithelium detachment. Am J Ophthalmol 2004; 138: 1077-1079.

25 Boscia F, Parodi MB, Furino C, Reibaldi M, Sborgia C. Photodynamic therapy with verteporfin for retinal angiomatous proliferation. Graefes Arch Clin Exp Ophthalmol 2006; 244: 1224-1232.

26 Montero JA, Ruiz-Moreno JM, Sanabria RM, FernandezMunoz M. Efficacy of intravitreal and periocular triamcinolone associated with photodynamic therapy to treat retinal angiomatous proliferation. Br J Ophthalmol 2009; 93: $166-170$. 